\section{Pädiatrische Palliativversorgung}

\section{Frühe Begleitung durchaus erwünscht}

Bei Erwachsenen umstritten, beginnen pädiatrische Palliativkonzepte (PPV) nicht erst mit der infausten Prognose. Bereits 1998 stellte die WHO fest, dass die aktive und ganzheitliche Betreuung mit dem Ziel der Lebensqualitätssteigerung bei Kindern bereits ab der Diagnose und auch bei einer Chance auf Heilung erfolgen soll. Dies geschieht selten. Als Grund wird u. a. das "they are not ready syndrome" angeführt. Die Studie mit 129 Kind-Eltern-Paaren bestätigte dies nicht.

Grundlage der Erhebung waren zwei neu entwickelte Fragebögen, die Kindern (10-17 Jahre) mit onkologischen Erkrankungen und ein Elternteil in unterschiedlichen Räumen gleichzeitig vorgelegt wurden. Die Patienten waren durchschnittlich 14 Jahre und der Elternteil 41,6 Jahre alt (überwiegend Mütter). Das Intervall zwischen Erstdiagnose und der Intervention betrug 1-12 Monate. Die häufigsten Diagnosen waren solide Tumoren, Leukämien,

Lymphome und Gehirntumoren. Körperliche und psychische Symptome waren häufig. Am stärksten litten die Kinder unter Übelkeit, Appetitverlust, Obstipation und Schmerzen, gefolgt von Angst und Depressionen. Die elterliche Einschätzung des Leidensdrucks wich davon teilweise ab (Konkordanz für die Symptome $40-69 \%$ ). $75 \%$ der Kinder und $69 \%$ der Eltern wünschten sich den Behandlungsschwerpunkt "Lebensqualität".
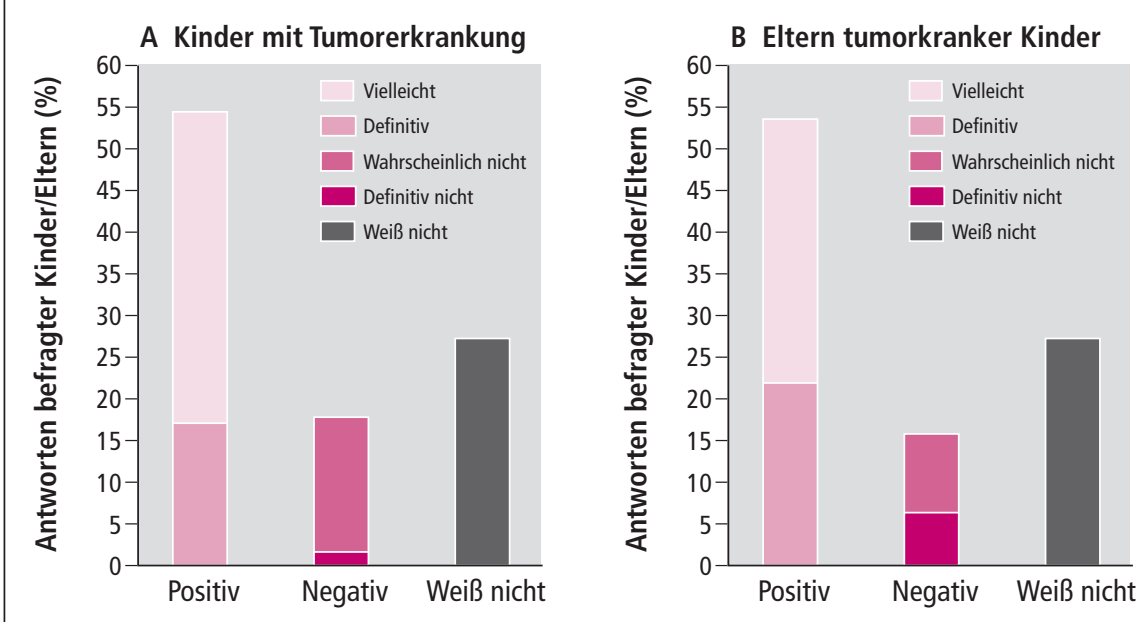

Abb. 1 Einstellung von Kindern mit Tumorerkrankung und ihren Eltern zu einer frühen Integration palliativer Versorgungskonzepte in die pädiatrische Onkologie (mod. nach [1])

\section{Palliativkonzepte kaum bekannt}

$98,4 \%$ der Patienten und $69,8 \%$ der Eltern hatten noch nie etwas von PPV gehört. Mehr als die Hälfte der Befragten stand der PPV definitiv/wahrscheinlich positiv gegenüber oder war noch unsicher. Eine negative Grundhaltung hatten wenige. Nur 1,6\% der Kinder und 6,2\% der Elternteile wünschten definitiv nicht, das Palliativteam bei der Erstdiagnose kennen zu lernen ( $>$ Abb. 1). Ungünstige Einflüsse auf die Beziehung zum Onkologen, Verlust von Hoffnung auf Heilung oder Therapieinterferenzen vermuteten die jungen Patienten und ihre Eltern selten.

Die Kinder waren besonders offen für die PPV und verbanden damit auch die Annahme einer Symptomlinderung (40,3\%; Eltern 17,8\%). Die Patienten hielten die PPV öfter hilfreich als ihre Eltern für das Schmerzmanagement, die onkologische Therapie und den gesamten Behandlungsverlauf. Mit der Kenntnis, dass die PPV auch die Sterbebegleitung einschließt, wollten etwa $15 \%$ der Kinder und Erwachsenen keine PPV ab der Erstdiagnose, während 26,4\% (Patienten) und $17,8 \%$ (Eltern) sie noch stärker begrüßten.

Insgesamt waren $58,9 \%$ (Patienten) und $50,4 \%$ (Eltern) für die frühzeitige Einbeziehung des Palliativteams. Besonders die Kinder mit einer subjektiv schlechten Lebensqualität begrüßten die Begleitung ab der Erstdiagnose $(73,1 \%)$. Die Autoren kommen zu dem Schluss, dass die jungen Patienten und ihre Eltern durchaus bereit für die frühe Palliation sind und empfehlen die Weiterentwicklung und Optimierung integrierter Therapiekonzepte, die ab der Erstdiagnose einsetzbar sind.

\section{Dr. med. Susanne Krome, Melle}

\section{Literatur}

1. Levine DR et al. Patients' and Parents' Needs, Attitudes, and Perceptions About Early Palliative Care Integration in Pediatric Oncology. JAMA Oncol 2017; 3: 1214-1220. 